\title{
Programas intergeneracionales y participación social: la integración de los adultos mayores españoles y latinoamericanos en la comunidad ${ }^{*}$
}

\section{Intergenerational Programs and Social Participation: The Integration of Spanish and Latin-American Seniors in the Community}

\author{
María de la Villa Moral JiméneZa \\ Universidad de Oviedo, España \\ ORCID: http://orcid.org/0000-0003-2421-9675
}

a Autor de correspondencia. Correo electrónico: mvilla@uniovi.es

Para citar este artículo: Moral Jiménez, M. de la V. (2017). Programas intergeneracionales y participación social: la integración de los adultos mayores españoles y latinoamericanos en la comunidad. Universitas Psychologica, 16(1), 1-19. http://dx.doi.org/10.11144/J averiana.upsy16-1.pips

\section{RESUMEN}

De acuerdo con una perspectiva de análisis contextual y dialéctica del transcurso de la vida, se ofrece un estudio psicosociológico de las relaciones intergeneracionales en contextos familiares. Se resaltan las similitudes y divergencias del proceso de envejecimiento en contextos europeos y latinoamericanos atendiendo al proceso de postmodernización de la familia. Se promueve el envejecimiento activo y la superación optimizante del paradigma de la decadencia y la pérdida, para lo cual se valora el fomento y la dinamización de la participación social y comunitaria de los adultos mayores en programas intergeneracionales. Se concluye que en nuestras sociedades, tanto del arco mediterráneo como latinoamericanas, la proliferación de redes de apoyo social basadas en el compromiso de la reciprocidad intrafamiliar e intergeneracional son un ejemplo del paradigma del envejecimiento productivo.

Palabras clave

envejecimiento activo; edad social; programas intergeneracionales; compromiso intrafamiliar.

\begin{abstract}
According to a dialectical context of lifetime analysis perspective, provides a psychosocial study of intergenerational relationships in familiar contexts. Emphasis is placed on the similarities and differences of the process of aging in European and Latin-American contexts attending to the process of post modernization of the family and to macro situational aspects. It also promotes active aging and optimizing overcoming the paradigm of decay and loss, for which values the development and revitalization of social and community participation of older people in intergenerational programs. We conclude that in our societies, the Mediterranean and Latin-America, the proliferation of social support networks based on the commitment of the reciprocity and inter generational are a clear example of the paradigm of the productive aging. Keywords active aging; social age; intergenerational programs; domestic commitment.
\end{abstract}


En el análisis del proceso de envejecimiento ha de considerarse la interdependencia entre aspectos psicosociológicos y psicoevolutivos ya que, en su estudio comprehensivo e integrador, constituyen áreas fenoménicamente interrelacionadas, máxime dada la implicación de condicionantes sociohistóricos moduladores del mismo (Bustillo, Fernández-Ballesteros, \& Huici, 2012; Domínguez, 2016; Fernández Ballesteros, Bustillos, Huici, \& Ribera, 2012). De este modo, en el proceso de construcción histórica de los objetos de estudio, basándose en intersubjetividades y acuerdos previos, se otorgan significados y formulan discursos que actúan como instrumentos de evaluación, control, valoración y reinterpretación de fenómenos como el envejecimiento, convirtiéndonos los investigadores sociales en unos agentes privilegiados de construcción de realidades normativizadas.

De acuerdo con una perspectiva de análisis contextual y dialéctica del transcurso de la vida, con la que mostramos nuestro acuerdo, se defiende la preeminencia de la interacción social como eje vertebrador de las relaciones humanas, en escenarios y contextos social y culturalmente determinados (véase Dulcey, 2010). En este sentido, se pretende comprender la experiencia y la conducta humana en virtud de los nexos reales, imaginarios y simbólicos que vinculan a los hombres entre sí en su devenir cotidiano, de ahí que, al fin y al cabo, desde perspectivas de análisis psicosociales, como la adoptada en este estudio, se pretende entender la condición humana, social y simbólica (Ibáñez, 1990, 1994). Las realidades del mundo actual se han ido volviendo cada vez más complejas, de manera que, dadas las interrelaciones e interconexiones de los constituyentes psicológicos, sociales, biológicos, económicos, políticos, culturales y ecológicos, ha se sugerirse una nueva manera de abordar comprehensivamente realidades multidimensionales a partir de una "lógica arborescente", en los términos de Morin (1983). Aplicada esta visión comprehensiva al desarrollo psicoevolutivo (véase Baltes, 1987, 1997, 2002) se promueve la necesidad de plantear enfoques psicosociológicos, culturales y sociocomunitarios del envejecimiento que faciliten la reflexión integradora, crítica y transformadora de modelos basados en concepciones normativas y desde los que no se prioricen estrictamente criterios de edad, dentro de estudios propios de la denominada postgerontología (Iacub, 2002; Moody, 2010) e incardinados en una conceptualización del progresivo proceso de postmodernización de las familias, en los términos de Meil (1999). En tales circunstancias propias de un contexto macrosituacional sometido a profundos cambios, el análisis integral de las relaciones intergeneracionales en contextos familiares y de sus implicaciones representa una cuestión de sumo interés para la Psicología Social (Antonucci, Birditt, Sherman, \& Trinh, 2011; Dulcey, 2010; Herrera, Sani, \& Bowe, 2011; Monserud, 2008; Triadó \& Osuna, 2005; Vila, 2011).

$\mathrm{Al}$ aproximarnos a las realidades psicosociológicas de los adultos mayores se ha de adoptar un posicionamiento riguroso y comprehensivo, desmitificador y crítico con análisis reduccionistas basados, por ejemplo, exclusivamente en criterios de edad. En conocida expresión de Pinillos (1992), la edad no es pura cronología, de modo que "no consiste solo en los años que se tienen, sino en lo que se hace con ellos".

Según la acción del tiempo físico y sus paradojas sobre diversos parámetros se pueden llegar a distinguir diversas edades: la edad cronológica (número de años), en avance irrefrenable: la edad biológica (organismo), con pérdidas y ganancias: la edad psicológica (personalidad) y, finalmente, con contactos y ausencias: la edad social (competencia social). Incluso se alude a la calificada como edad funcional que trata de medir la capacidad del individuo para desenvolverse en el medio social comunitario (Guillén, 1992). Pues bien, en la edad social y funcional nos centraremos, de manera que aun siendo cierto, en buena medida, que el chronos físico deteriora, el tempus social enriquece. De este modo, aunque la edad se contempla como un criterio para definir la vejez, otras variables podrían ser más 
definitorias, tales como el nivel de salud (o envejecimiento primario) o el estilo de vida (o envejecimiento secundario) (Izquierdo, 2008) (véase OMS, 2015). La diversidad, en el tiempo y en el espacio, de la duración media de la vida humana, no permite delimitar la vejez en términos de años, cuando se trata de trazar su historia y su porvenir., sino que debe hacerse en términos de potencialidades, funciones y situación social, modificando representaciones colectivas y actitudes individuales. Así es como ha de ser evaluada la aventura de envejecer, calificada así por Pàmies (2002). Lo que podría llamarse edad social depende de la conjunción de distintos factores: la longevidad en el momento y lugar dados, la función que la sociedad atribuye a las personas que considera jóvenes o maduras y la que asigna a las califica de viejas. Se aboga por la inclusión de factores sociopsicológicos desde una perspectiva como la aportada en los estudios de Riegel y Meachan (1976a, 1976b). Así, las actitudes y representaciones colectivas hacen viejos a nuestros mayores (Moral, 1999), de ahí la necesidad de modificarlas para favorecer la necesidad de repensar el envejecimiento activo, envejecimiento en positivo (véase Alonso \& Mackay, 2011; Herce, 2016; Llorente, 2012; Rodríguez, 2009). Baste recordar que desde la teoría de la actividad se establece que los adultos mayores deben continuar con unos niveles de actividad y participación óptimos en orden de alcanzar el bienestar psíquico y social, como garante de una vejez competente e integrada (Fernández-Ballesteros, 2008, 2009a, 2009b; Fernández-Ballesteros, Molina, Schettini, \& Santacreu, 2013).

La equiparación de la vejez a multitud de déficits, deterioros o pérdidas de índole físico, psicológico o social presenta una inusitada raigambre en la historia y el saber popular, tal y como fundamentó Minois (1989), así como existe suficiente evidencia empírica que confirma el hecho de que sobre la vejez se siguen manteniendo creencias, actitudes y estereotipos negativos (Carbajo, 2009; Sánchez, Trianes, \& Blanca, 2009) que abundan en su interpretación peyorativa mediante la que se incide en el deterioro biológico, psicológico y sociomunitario del adulto mayor y que han de ser modificados (véase Melero, 2007). Resulta llamativo que, a pesar de que va aumentando el promedio de vida, nuestras expectativas culturales acerca del envejecimiento, no vayan en consonancia con tales progresos (Rice, Löckenhoff, \& Carstensen, 2002). Tradicionalmente, la vejez ha sido valorada dicotómicamente o bien mediante una valoración negativa, que equipara vejez con estado deficitario o bien mediante una consideración positiva de forma excluyente, visión que también es mitificada. Ambas visiones dificultan el análisis comprehensivo e integral de esta condición y limitan una optimizante integración del adulto mayor en la sociedad, así como la valoración de su potencial.

Expuesto lo anterior, nuestro objetivo es recontextualizar adecuadamente la vejez desde la perspectiva del envejecimiento activo y reinterpretarla desde posicionamientos psicosociales críticos, con sus pérdidas y ganancias, sus declives y optimizaciones. Asimismo, se plantea la necesidad de abordar comprehensivamente el papel de los adultos mayores en la familia y su participación social en la comunidad como red de apoyo, a modo de alianza intergeneracional.

\section{Planteamiento}

\section{Mayores, programas intergeneracionales $y$ participación social}

El hombre es un ser eminentemente social y relacional, dado que vive su "realidad" psicosocial vinculada e interpretada en función de su vinculación a los otros. No es, pues, un individuo aislado, sino un todo integrado bio-psico-social en un entorno con el que establece una retroalimentación dinámica, ya que no actúa como un agente meramente pasivo, sino en constante interacción con su contexto sociocultural y enraizado en el imaginario colectivo. De acuerdo con esta perspectiva de análisis comprehensiva e integradora, se incide en la idea relativa a la construcción social de la vejez (véase Fernández \& Kelh, 2001; Moral, 
1999; Ruiz, Scipioni, \& Lentini, 2008). Se parte de una explicación de los fenómenos sociales basada en la potenciación de su desarrollo a partir de contextos sociales en los que su cotidianeidad está determinada por la cultura, la historia y el contexto social. Semejante orientación construccionista es deudora de los planteamientos del interaccionismo simbólico y la fenomenología de Alfred Schütz, como lo es también de Berger y Luckman, especialmente de su obra La construcción social de la realidad (1966), donde explican cómo a través de la interacción social se crean realidades objetivas de diferente orden y cómo estas son internalizadas a través del proceso de socialización. Se valora la realidad social como construida, en proceso dinámico, y reproducida por quienes actúan a través de sus interpretaciones y de su conocimiento. El sujeto va creando significados, nuestro mundo, lo cual constituye una exteriorización, de la que resultan unos productos objetivos, los cuales vamos internalizando, de manera que se trata, en suma, de un proceso dialéctico, de naturaleza social, a través del cual se va construyendo la realidad. De este modo, las personas desean tener sus propias interpretaciones de los acontecimientos, de ahí sus intentos de justificar o garantizar sus versiones (Gergen, 1982, 1991).

Como animales sociales que somos - de acuerdo con la máxima aristotélica, retomada en el texto homónimo de Aronson (1985)-, las relaciones humanas no se dan en un vacío social, sino que nacemos, nos desarrollamos, vivimos y morimos vinculados a los demás, física y emocionalmente. Para nuestra salud socioafectiva es imprescindible gozar de unas adecuadas relaciones interpersonales a cualquier edad, de manera que el disponer de una red de apoyo y vinculación social y comunitaria constituye una necesidad psicosocial (véase Neri, 2007). Todos los seres humanos precisamos de una serie de interacciones personales mediante las cuales conseguimos el apoyo socioafectivo, emocional o material mediante cuyos mecanismos de acción logramos superar momentos de dificultad o abatimiento. En este sentido, se ha constatado que el aislamiento social durante la vejez empeora el deterioro cognitivo, conductual e inmunitario (Arranz, Giménez-Llort, De Castro, Baeza, \& De la Fuente, 2009). En contrapartida, los apoyos materiales, informacionales e instrumentales son básicos y constituyen, los tres tipos de apoyos brindados por las redes sociales mediante los cuales se tienden a cubrir nuestras necesidades y déficits en diversos terrenos socioafectivos y de resolución de problemas. Ciertamente, los procesos psicosociales implícitos en cualquier intercambio interactivo durante toda la vida (niñez, juventud, adultez y vejez) desarrollan la sociabilidad (Caamaño \& Martínez, 2012), así como la sensibilización y participación comunitaria, también en el estado de transición a la vejez que ha de verse dignificado por la recuperación del renovado rol social de los adultos mayores (véase Martínez del Castillo, Jiménez-Beatty, Graupera, \& Rodríguez, 2006).

El envejecimiento es un hecho que afecta a toda la población mundial, y de manera considerable a los países más desarrollados, en los que la esperanza de vida ha ido aumentando de manera considerable, tal y como se refleja en el informe de Naciones Unidas (2014). De este modo, aunque el fenómeno está más avanzado en Europa y en América del Norte, el envejecimiento de la población se está produciendo en otras muchas regiones principales del mundo. Así, a escala mundial, la proporción de personas mayores (de 60 años o edad superior) aumentó del $9 \%$ en 1994 al $12 \%$ en 2014, y se espera que alcance el $21 \%$ en 2050.

Por lo que respecta a la Unión Europea España se sitúa en la quinta posición de los países más envejecidos, según los datos de Eurostat (2012, 2014). Si hacemos una previsión del envejecimiento de la población en el mundo para el año 2050, las cifras de Naciones Unidas (2014) sitúan a España como el segundo país más envejecido del mundo. Los datos actuales muestran que en el caso español el fenómeno de envejecimiento se observa especialmente acelerado ya que en menos de 30 años se ha duplicado el número de personas mayores de 65 años. Esta posición se debe, básicamente, al rápido e importante retroceso de la fecundidad, cuyo nivel se encuentra entre los más bajos 
del mundo. De este modo, en España la tendencia al envejecimiento es mayor que en el resto de Europa, pues se prevé que en 2050 los mayores de sesenta años representen el $38.3 \%$, esto es, cuatro puntos por encima del promedio europeo. El sobreenvejecimiento tendrá, y está teniendo ya, consecuencias diversas, una de ellas es que cada vez habrá más adultos mayores dependientes. Si para el citado 2050 se prevé un aumento considerable de población octogenaria, la resolución y apoyo de las situaciones de dependencia deberá ser un elemento indispensable de la sociedad futura (I.N.E., 2011). Estos datos reflejan el fenómeno conocido como transición demográfica, mediante el que se incide en los descensos tanto en tasas de natalidad como de mortalidad, así se constata que las personas mayores son el grupo de población de más rápido crecimiento en el mundo, pues en términos absolutos, el número de personas mayores de 60 años casi se ha duplicado entre 1994 y 2014 y, específicamente, el porcentaje mundial de personas mayores de 80 años era del $14 \%$ en 2014, y se espera que ascienda al $19 \%$ en 2050, lo cual supone que en 2050 habría 392 millones de personas mayores de 80 años, es decir, más de tres veces que en la actualidad (Naciones Unidas, 2014). Semejantes prospectivas representan un reto a nivel mundial y para la Comisión Europea, tal y como se refleja en su informe sobre demografía bajo el ilustrativo título Meeting social needs in an Ageing society (2008). Así, de acuerdo con los datos contenidos en el informe Eurostat (2014), la estructura y el perfil de la población de la UE-28 han cambiado considerablemente, de modo que entre las iniciativas emblemáticas de la Estrategia Europea 2020 se aborda el envejecimiento demográfico como auténtico desafío.

De acuerdo con las prospectivas demográficas del Instituto Nacional de Estadística (INE, 2011) en España se plantea una intensidad del proceso de envejecimiento de la población residente en España, acelerado por un descenso de la natalidad que no llega a verse compensado con el saldo migratorio positivo. Se estima que el porcentaje de población mayor de 65 años, que actualmente se sitúa en el $18.2 \%$ pasaría a ser el $24.9 \%$ en 2029 y del $38.7 \%$ en 2064 . Atendiendo a los indicadores estadísticos básicos contenidos en el perfil de las personas mayores en España, expuestos por Abellán y Pujol (2015) se concreta que España, junto con países como Alemania, Italia, Francia y Reino Unido son los países de la Unión Europea con una cifra más alta de personas mayores, de modo que específicamente a los 65 años, la esperanza de vida de hombres y mujeres en España es de las más altas de la UE, detrás de Francia. A este proceso de envejecimiento de la población española se asocian múltiples implicaciones en los planos laboral, sanitario y socioeconómico (véase Igartua, 2016) vinculados a la necesidad de renunciar a la tiránica barrera psicológica de los 65 años, en los términos de Herce (2016).

En el contexto latinoamericano se vive un envejecimiento moderado (Dulcey, 2010, 2011; Dulcey, Izquierdo, \& Franco, 2008), siendo el porcentaje de mayores de 60 años algo superior al $9 \%$. La tasa de crecimiento de dicha población entre los años 2000 y 2025 se calcula, en promedio en $3.4 \%$. (CEPAL, 2007; Population Reference Bureau, 2007, 2009; Uprimny, 2010). En América Latina y el Caribe, entre los años 2000 y 2050, el porcentaje de personas de 60 y más años se triplicará (25.5\%, en el 2050), de modo que se prevé que, para el 2050, uno de cada cuatro habitantes de la región será mayor de 60 años (Naciones Unidas-CEPAL, 2009, 2014). En el informe de previsiones demográficas realizado por el CELADE-División de Población de la CEPAL (publicado en el Observatorio demográfico № 11, 2011), se apunta que un aspecto destacado de los cambios demográficos observados y proyectados para América Latina es la reestructuración etaria de la población. Según las previsiones, en América Latina el número de adultos mayores superará por primera vez al de niños en 2040, de manera que cobrará un mayor peso relativo en el total de la población, al pasar de apenas un $5.6 \%$ del total de la región en 1950 a un $9.9 \%$ en 2010 y proyectándose que en 2100 representará más del $35 \%$ de la población (CEPAL, 2008, 2011; Naciones Unidas, 2010, 2011). De este modo, en el estudio comparativo sobre envejecimiento 
demográfico de López-Calleja y Morejón (2015), se estima que en Cuba, Chile, Argentina y Panamá el índice de envejecimiento alcanza los valores más elevados. En tales contextos abundan las transferencias intergeneracionales en hogares donde se posibilita una mayor coexistencia de generaciones en las familias actuales, a modo de verticalización de la familia (Cabré, 2000).

Dado nuestro interés específico en las relaciones sociales e intergeneracionales durante la (mal) llamada tercera edad — aparte de la constatación de la trascendencia a múltiples niveles del apoyo social, la estabilidad afectiva y emocional que actúan como factores protectores - se precisa intervenir para desmitificar mitos y prejuicios asociados a objeto de que, parafraseando a Pinillos (1992), la llamada tercera edad deje de ser una edad de tercera.

\section{Programas intergeneracionales como capital social y comunitario}

Descritas la situación y prospectivas sociodemográficas, es incuestionable el potencial productivo, fundamentalmente a nivel psicosociológico, de estos adultos mayores con gran valía y experiencia, de ahí la importancia concedida a los contactos sociales formales e informales, ya sea en redes interactivas o interacciones puntuales, insertas en programas intergeneracionales o mediante la potenciación de participaciones activas de los mayores en la comunidad. Es un hecho constatado que las redes sociales de apoyo contribuyen a conformar y reafirmar nuestra identidad personal y social (Gracia \& Herrero, 2005; Pinheiro, Koller, \& Sánchez-Soares, 2008). Sin embargo, tiende a aludirse, estereotípicamente, a la idea de pérdida y abandono de relaciones sociales y al escaso apoyo social brindado a los adultos mayores dado que sus redes y contactos sociales se consideran reducidas a mínimos. La constatación de la existencia de una fuerte asociación entre edad y apoyo social se evidencia en los análisis correlacionales efectuados hace más de dos décadas por Fernández-Ballesteros y su equipo (1992) los cuales confirman que, si bien a partir de los sesenta y cinco años se produce un decremento significativo de este parámetro relacional, también se constata el potencial papel protector que las relaciones interpersonales ejercen sobre la salud, pues el apoyo psicosocial brindado a cualquier individuo se convierte en un mecanismo que facilita el afrontamiento de eventos estresantes. Desde la perspectiva de envejecimiento productivo, propuesto en la literatura gerontológica por Butler (2000), debe entenderse como "la capacidad de un individuo o una población para servir en la fuerza de trabajo remunerada, en actividades de voluntariado, ayudar en la familia y mantenerse independiente como sea posible" (p. 6).

Los programas intergeneracionales y las labores asociativas, con todo aquello que representan a nivel socioafectivo, suponen un ejemplo inequívoco de entrega e interacción humana. Baste recordar a este respecto que el año 2012 fue declarado por el Parlamento y la Comisión Europea como el Año Europeo del envejecimiento activo y la solidaridad intergeneracional. En consonancia con lo expresado hace décadas por Ibáñez (1985), se aboga por la necesidad de reintegrar el tejido social como un aspecto insoslayable de cualquier planteamiento optimizador de esta etapa vital o de cualquier otra. Al respecto, según el Consorcio Internacional para los Programas Intergeneracionales, un programa intergeneracional, es un medio para el intercambio concreto y continuado de recursos y aprendizajes entre las generaciones mayores y las más jóvenes con el fin último de conseguir beneficios individuales y sociales. Los programas intergeneracionales constituyen una metodología de acción social que actúa en la comunidad sobre las bases de un modelo de solidaridad intergeneracional (Pinazo, 2012). Tales programas se incardinan en planes de acción planteados a dos niveles de desarrollo: individual y social. En el primer nivel, los programas que se basan en la iniciativa, intereses y motivos de individuos concretos, aprovechan los recursos humanos, potencian las relaciones naturales de apoyo a la vecindad y el valor añadido de cada generación. La solución de 
problemas sociales relevantes constituye la base de los programas que se basan más en una planificación institucional, maximizando sus recursos y otorgando oportunidades a las comunidades locales para el diseño e implementación de programas ajustadas a los recursos y necesidades. Romper el aislamiento generacional, así como dar oportunidad de voluntariado a todas las edades, construyendo redes de apoyo social en la comunidad constituye un apremiante reto. De este modo, la potenciación del intercambio entre las generaciones en la comunidad contribuye a la reducción de la visión negativista sobre el proceso de envejecimiento, favoreciendo el entendimiento y el progreso social, así como se erigen en una herramienta de reivindicación y transformación social y apoyo a la ciudadanía. Para cada individuo que participa de ellos representan proyectos motivados por el deseo de compartir esfuerzos e ilusiones. Ciertamente, los programas intergeneracionales son un vehículo que promueve la solidaridad intergeneracional en la comunidad, lo cual genera nuevos sistemas de apoyo, representa un elemento que favorece la convivencia social y proporciona ayuda en la resolución de conflictos sociales (véase LópezPastor, 2014; Sancho, Tomás, Gutiérrez, Oliver, \& Galiana, 2015).

Cultivar las relaciones intergeneracionales y la participación en la comunidad constituye un modalidad de vinculación de efectos muy positivos ya que la existencia de unos círculos de contacto, asociación, amistad y cooperación disminuyen los posibles sentimientos de soledad, al tiempo que afianzan la autoestima y la identidad personal y social de la persona mayor, la cual se siente apreciada e integrada en una red social que le brinda apoyo. Inequívocamente se conviene en la necesidad de crear comunidades para todas las edades, promoviendo la interrelación de niños, jóvenes, adultos y adultos mayores, máxime dada la interdependencia entre las mismas (Bressler, Henkin, \& Adler, 2005), de acuerdo con el concepto de comunidad de generaciones. De este modo, dado el interés en promover un conocimiento de las modalidades de vinculación comunicativa intergeneracional y de sus limitaciones y fortalezas, que pueden incrementar estereotípicamente las percepciones de diferenciación y la consiguiente posible segregación entre unos y otros, se ha investigado sobre las formas de comunicación entre individuos de diversos espectros de edad, incidiéndose particularmente en los estratos poblacionales de jóvenes y mayores con estudios en contextos anglosajones (véase Harwood, Mckee, \& Mei-Chen, 2000; Williams \& Garret, 2002) o bien con análisis de la comunicación intergeneracional en el contexto latinoamericano, tales como con población costarricense (Pérez \& Smith, 2008).

La coexistencia e interacción entre diversas generaciones fomenta los lazos de interdependencia y de apoyo social, cuestión de trascendental importancia para la satisfacción de unas necesidades de contacto, interacción e intercambio que conforman una identidad, un concepto de sí mismo y unos mecanismos de afrontamiento de eventos estresantes, así como una satisfacción vital, como beneficios suficientemente constatados por la literatura sobre el tema (López-Pastor, 2014; Sancho et al., 2015). Asimismo, repercute beneficiosamente sobre los propios jóvenes que se nutren de experiencias que favorecen su proceso de socialización. Baste recordar que desde teorías como las del intercambio se alude a un principio optimizador de nuestros recursos, al hecho de que todas las generaciones que coexisten en un momento determinado constituyen la estructura social y puede contribuir y recibir algo del resto de las generaciones (véase Albuerne \& Juanco, 2002; Delgado, Calero, \& González, 2016; García, García, \& Nieto, 2013; Padrós, Moll, \& Gancedo, 2009). La constatación de beneficios sobre el desarrollo cognitivo, afectivo y social es evidente. La interdependencia entre generaciones vigoriza el componente socioafectivo implicado en los procesos interactivos. Podría incidirse en el mecanismo de compensación prosocial intrafamiliar ya que la red familiar funciona como capital relacional de reserva, en los términos de Meil (2004), que puede activarse para resolver los 
cambios y dificultades a los que se tiene que hacer frente en el curso de la vida.

Semejante vinculación en condiciones como las actuales es calificada por algunos como solidaridad familiar (Bazo, 2008, 2012) como base de un aprendizaje y reestablecimiento de un pacto generacional en condiciones de interacción y/o convivencia (Barreto, Pérez, Molero, \& Gómez, 2011; Galindo, 2008; Gómez, 2012) y como potenciación de la cohesión social vía solidaridad intergeneracional (Hatton-Yeo, 2007). En cambio, según Goñi (2009), tales vínculos pueden conducir a una suerte de despotismo ilustrado familiar en una sociedad ambivalente con la asunción de una excesiva responsabilidad y potestad sobre la adopción de decisiones en adultos mayores frágiles. Idealmente, la relación entre miembros de diferentes generaciones potencia el sentimiento de solidaridad de la sociedad y representa un garante no solo de la transmisión del pasado que nos pertenece a todos, sino de anticipación de un pasado mañana, del que también todos hemos de ser partícipes.

Desde una perspectiva psicosocial se proclama que el desarrollo de la sociabilidad a través de las agencias socializadoras por excelencia se completa con la acción sociocomunitaria. La canalización del apoyo social se produce a través de redes sociales y contactos interpersonales, ya sea entre los miembros de una misma cohorte de edad o de forma intergeneracional. En este último caso, estaríamos aludiendo a la construcción de redes de apoyo social en la comunidad, de manera que el impacto de estos programas desde los cuales se atiende a la interdependencia entre generaciones se manifiesta de forma individual sobre el cambio de actitudes, el incremento de la calidad de vida y el ajuste personal junto con el sentimiento de utilidad y autosatisfacción. Asimismo, el control sobre la propia vida, en términos de autonomía personal y autoeficacia constituye un importante indicador de calidad de vida, tal y como se ha demostrado en población de adultos mayores colombianos (Dulcey, Mantilla, García, \& Alfonso, 1998; Trujillo, 2005; Trujillo, Tovar, \& Lozano, 2007).
Se defiende que el fomento y la dinamización en la participación social de los adultos mayores representan una labor de intervención sociocomunitaria de suma importancia a múltiples niveles. La significación de sus labores de participación social es doble, individual y colectiva. Desde una perspectiva individual, es una de las estrategias que se derivan del concepto ya citado de envejecimiento activo, mediante el cual se trata de optimizar recursos para una vejez exitosa, promoviendo la salud individual, afrontando el propio proceso de envejecimiento mediante la adopción de un rol activo y sin desconectarse o aislarse de las vinculaciones intergeneracionales y de la propia comunidad. Por otro lado, desde una dimensión colectiva, la participación asociativa de los adultos mayores beneficia a la propia sociedad, contribuyendo al equilibrio social y comunitario, así como dinamiza la dimensión cooperativa y prosocial y propiamente la reivindicativa.

Teniendo en cuenta los principios de independencia, participación, cuidados, autorrealización y dignidad en acciones y programas destinados a los adultos mayores (ONU, 2010, 2011) una labor fundamental en esta dirección es promocionar los derechos de ciudadanía, con especial énfasis en aquellos relacionados con la participación social a través de la educación permanente, tales como la enriquecedora participación en programas educativos universitarios para adultos mayores (Fernández, García, \& Pérez, 2014; Lucas, Pérez, Fonseca, \& Ortuño, 2015; Molina, Martínez, Bordes, López, \& Piñero, 2015; Rodríguez, Gutiérrez, \& Seijas, 2012). De este modo, los adultos mayores representan un potencial social y asociativo que hemos de valorar y apoyar en su compromiso de participación.

Dada la mayor sensibilización, no solo institucional, sino también a nivel sociocomunitario, se ha constatado en los últimos años una proliferación de programas en los que se incide en las ventajas integrales de los encuentros intergeneracionales incardinados dentro de una filosofía optimizante de promoción de vida activa en los adultos mayores. Abundan los programas desde los que se promueve su acercamiento 
a los cambios culturales y sociales que tan aceleradamente vivimos, dinamizando centros y residencias con el ambiente activo y positivo que transmiten jóvenes y niños, fomentando, asimismo, la integración de residencias geriátricas en la sociedad (Cobo \& Codina, 2008; Serrani, 2010), ya sea tratando de paliar los efectos del aislamiento de la población mayor rural con programas adecuados (Aristegui, Cuenca, Ahedo, Cuenca, \& Monteagudo, 2015; González, 2010) o bien, desde perspectivas más integrales, mediante la potenciación de debates intergeneracionales sobre problemáticas de gran calado social, tales como la violencia de género (González, Pedrero, Pérez, \& Prieto, 2008). Asimismo, se asiste a una revitalización del interés por los programas que involucran activamente a abuelos y nietos en programas en los que se potencia el interés por las propias vinculaciones y por la recuperación de juegos y costumbres tradicionales (véase Llaneza, 2010; Pinazo \& Montoso, 2004; Romero, Serrano, \& Godoy, 2015; Ruiz \& Vicente, 2006; Sánchez, Buz, Bueno, \& Navarro, 2007). Por otra parte, el voluntariado social de los adultos mayores constituye un auténtico motor de creación y fortalecimiento de redes intergeneracionales basado en la transmisión de la experiencia como estrategia de inclusión social y de movilización solidaria de recursos humanos de gran valor (véase Fernández, 2012).

Expuesto lo anterior, se valora que la ampliación del capital social y colectivo, así como de la potenciación de un pacto solidario entre generaciones que contribuya a la redefinición de los derechos y los deberes ciudadanos de todos los grupos de edad constituye un reto inexcusable en las sociedades contemporáneas.

\section{Conclusiones}

Vivimos tiempos de profundos cambios a múltiples niveles, de desregulaciones varias y de grandes potencialidades. A nivel psicosociológico se están experimentando también modificaciones en los procesos de vinculación interpersonal, tal y como se constata en la literatura sobre el tema
(Alonso \& Mackay, 2011; Funes, 2011; Scheibe \& Carstensen, 2010; Stenner, McFarquhar, \& Bowling, 2011). Se plantean nuevos retos desde una perspectiva psicosociológica vinculada al estudio del desarrollo, superadores de un edadismo asociado a estereotipos y prejuicios consolidados social y culturalmente, como mecanismo legitimador de desigualdad (Lemus \& Expósito, 2005; Moral, 1999). A otros niveles se constatan los cambios de transición demográfica en la población mundial (véase Naciones Unidas, 2014). Se constata un creciente proceso de modernización social de la vejez, de acuerdo con el análisis de López Doblas y Díaz (2013), abundándose en la idea de destradicionalización y cambio social (Sánchez-Oro, Pérez, \& Moreno, 2013; Sánchez-Vera, Algado, Centelles, López, \& Jiménez, 2009). Este hecho diferencial afecta a las sociedades europeas y latinoamericanas, lo cual ha de ser reinterpretado desde una perspectiva de análisis sociocultural, atendiendo a sus idiosincrasias e incardinando propuestas de intervención adecuadas a sus singulares contextos, si bien, con las salvedades apuntadas, ambas sociedades asisten a un progresivo proceso de envejecimiento, más evidente en las primeras. A pesar de la tendencia actitudinal a la juvenalización de la sociedad (Moral \& Ovejero, 2004), se va consolidando una sociedad envejecida y se agudiza la calificada como crisis de madurez y madurez en crisis (Gil Calvo, 2010), a pesar de lo cual ha de promoverse el proceso integral de ir envejeciendo sin ser viejo, en los términos de Ander-Egg (2010).

Ciertamente, la sociabilidad no entiende de edades, pero los estereotipos acerca de la condición del hombre y sus edades se amparan en una larga tradición que, de un modo u otro, como en una condición entrópica, nos persuade de sus supuestas "verdades". Con tantas crisis y desregulaciones como hay, resulta absurdo que algunos actualicen sempiternos conflictos intergeneracionales. Más bien, hemos de recuperar la dignificación de la vejez, no como renovada gerontocracia, sino en la esencia de lo humano, en las oportunidades asociativas y de vinculación que nos hacen realmente personas. 
En suma, en nuestras sociedades, tanto en el arco mediterráneo como latinoamericanas, la proliferación de redes de apoyo social basadas en el compromiso de la reciprocidad intrafamiliar son un ejemplo inequívoco del paradigma del envejecimiento productivo (Fernández-Ballesteros et al., 2013; Miralles, 2010) y suponen un recurso de inigualable valor que revitaliza lazos socioafectivos y que representa una modalidad en alza de solidaridad intergeneracional. Como se constata en el estudio de Hank (2012) sobre las relaciones entre generaciones en Europa o en estudios como los de la Encuesta Europea Share se valora que el papel de los adultos mayores se ha visto reforzado en el actual contexto de crisis socioeconómica, ejemplo inequívoco de lo cual es el aumento de las relaciones intergeneracionales en la familia, por ejemplo en labores de coalición familiar en tareas de responsabilización del cuidado de los nietos y por su colaboración económica (véase Romero et al., 2015). A este respecto, en el citado Informe de Naciones Unidas (2014) sobre el envejecimiento en el siglo XXI es calificado como una celebración y como un desafío. En este sentido, dada la necesidad de repensar el llamado estado del bienestar se asegura que la sostenibilidad del bienestar intergeneracional en Europa y la OCDE (véase G. López, , 2012) pasa por alianzas intergeneracionales. Baste recordar que la Unión Europea cuenta con las perspectivas de envejecimiento más importantes para los próximos años, lo cual afectará al modelo y a las propias políticas de bienestar, para lo cual grandes estrategias de acción, como la Estrategia 2020, asumen este desafío (Alonso \& Mackay, 2011).

En el contexto latinoamericano en el Documento sobre Personas Mayores, Dependencia y Servicios Sociales en los países del Cono Sur (OISS, 2008) se explicita que ha de contribuirse a la mejora de la calidad de vida de la población a través del desarrollo de los dispositivos de protección social y de atención de las situaciones de dependencia, así como potenciar la infraestructura de recursos sociosanitarios, además de considerar las redes de apoyo social y la participación de los adultos mayores en el movimiento asociativo y sus vinculaciones intergeneracionales. Así, según la CEPAL (2011), en América Latina y el Caribe en términos porcentuales, los adultos mayores respecto a la población total pasarán del $8 \%$ en el año 2000 al $14 \%$ en 2025, alcanzando un 23,4 $\%$ en el año 2050. Más en concreto, en los cinco países del Cono Sur que participaron en el estudio (Argentina, Brasil, Chile, Paraguay y Uruguay) viven la mitad de los adultos mayores de 60 años de toda América Latina y el Caribe. El interés en las convergencias y divergencias del proceso de envejecimiento entre los distintos países del Cono Sur y respecto a América Latina y el Caribe en su conjunto, con el objetivo de profundizar en el análisis de una visión panorámica que permita entender los rasgos comunes y diferenciales del envejecimiento de la región, es sumamente pertinente.

Indudablemente, el envejecimiento demográfico implica múltiples desafíos tanto para países de América Latina que se encuentran en etapas intermedias y avanzadas de la transición demográfica como en España, que presenta una transición demográfica avanzada (Garay \& Hebrero, 2015). Así, en el estudio comparado en América Latina y el Caribe realizado por Huenchuan (2016) se identifican las oportunidades y los desafíos planteados, así como la contribución de la agenda internacional a la institucionalización de los asuntos del envejecimiento y las propuestas sobre institucionalidad pública en la región. La formulación de una política integral hacia los adultos mayores en contextos latinoamericanos provee de un análisis integral de las necesidades en todos los planos de la vida individual y colectiva, así como de las transferencias familiares de apoyo económico, instrumental y emocional asociadas a las relaciones intergeneracionales y al empoderamiento de los adultos mayores (B. López, 2012) dentro de un modelo de envejecimiento dinámico (Jones, 2016). De este modo, según CEPALCELADE (2016), mediante los avances del Grupo de trabajo de Composición Abierta sobre el Envejecimiento de las Naciones Unidas y de la Convención Interamericana sobre la Protección de los Derechos Humanos de las Personas 
Mayores se incide en las buenas prácticas en materia de envejecimiento.

En definitiva, ha de promoverse activamente la creación de redes psicosociales para el intercambio del diálogo intergeneracional desde perspectivas de envejecimiento activo y prácticas de visibilización y empoderamiento mediante las que se contribuya a revitalizar la enriquecedora labor de los adultos mayores como proveedores de conocimientos, cuidados y capital humano (International Longevity Centre Brazil ILC-Br, 2015; Pérez Serrano, 2012; Petretto et al., 2016; Pinazo \& Poveda, 2016). Ciertamente, son las relaciones humanas solidarias, los intercambios socioafectivos, la participación social y comunitaria, y tantos otros procesos psicosociales enriquecedores, los que contribuyen a llenar nuestras vidas, en todo grupo etario, de modo que hemos de esforzarnos no en poner barreras, sino en trazar puentes.

\section{Referencias}

Abellán, A., \& Pujol, R. (2015). Un perfil de las personas mayores en España, 2015. Indicadores estadísticos básicos. Madrid: Consejo Superior de Investigaciones Científicas (CSIC). Centro de Ciencias Humanas y Sociales (CCHS). Recuperado de https://envejecimientoenred.wordpress. com/2015/01/26/el-estado-de-la-poblacion -mayor-en-espana-2015/

Albuerne, F., \& Juanco, A. (2002). Intergeneracionalidad y escuela: "Trabajamos juntos, aprendemos juntos". Revista Interuniversitaria de Formación del Profesorado, 45, 77-88. Recuperado de http ://www.redalyc.org/html/274/27404507/

Alonso, F., \& Mackay, M. (2011). Hacia una nueva cultura de ser mayor: cambios y oportunidades al filo del 2012, Año Europeo del Envejecimiento Activo y la Solidaridad Intergeneracional. Sesenta y más, 304, 8-13. Recuperado de https://dialnet.unirioja.es/servlet/articul $\mathrm{o}$ ? codigo $=3732629$
Ander-Egg, E. (2010). Cómo envejecer sin ser viejo. Madrid: CCS.

Antonucci, T. C., Birditt, K. S., Sherman, C. W., \& Trinh, S. (2011). Stability and change in the intergenerational family: a convoy approach. Ageing and Society, 31 (7), 1084-1106. https://doi.org/10.1017/S01446 86X1000098X

Aristegui, I., Cuenca, M., Ahedo, R., Cuenca, J., \& Monteagudo, M. J. (2015). La relación entre el entorno en el que viven las personas mayores en España y su acceso al ocio. Un análisis descriptivo. Inguaruak: revista vasca de Sociología y Ciencia Política, 59, 145-156.

Aronson, E. (1985). El animal social: Introducción a la Psicología Social. Madrid: Alianza.

Arranz, L., Giménez-Llort, L., De Castro, N. M., Baeza, I., \& De la Fuente, M. (2009). El aislamiento social durante la vejez empeora el deterioro cognitivo, conductual e inmunitario. Revista Española de Geriatría y Gerontología: Órgano oficial de la Sociedad Española de Geriatría y Gerontología, 44(3), 137-142. http://dx.doi.org/10.1016/j.regg.2 008.12 .001

Baltes, P. B. (1987). Theoretical propositions of lifespan developmental psychology: On the dynamics between growth and decline. Developmental Psychology, 23(5), 611-626. Recuperado de http://ibrary.mpib-berlin.m pg.de/ft/pb/pb_theoretical_1987.pdf

Baltes, P. B. (1997). "On the incomplete architecture of human ontogeny: Selection, optimization, and compensation as foundation of developmental theory". American Psychologist, 52 (4), 366-380. http ://dx.doi.org/10.1037/0003-066X.52.4.366

Baltes, P. B. (2002). Autobiographical reflections: from developmental methodology and lifespan psychology to gerontology. In J. E. Birren \& J. J. F. Schroots (Eds.), A History of geropsychology in autobiography (pp. 7-26). Washington DC, EE.UU.: American Psychological Association.

Barreto, M. P., Pérez, M., Molero, M., \& Gómez, M. J. (2011). Bienestar y convivencia intergeneracional: necesidades y recursos del anciano y su familia. Apuntes de 
Psicología, 29(1), 107-121. Recuperado de http://www.apuntesdepsicologia.es/inde x.php/revista/article/view/190

Bazo, M. T. (2008). Personas mayores y solidaridad familiar. Política y Sociedad, 45(2), 73-85.

Bazo, M. T. (2012). Relaciones familiares y solidaridad intergeneracional en las nuevas sociedades envejecidas. Panorama Social, 15, 127-142.

Berger, P. \& Luckman, T. (1966). The social construction of reality: a treatise in the sociology of knowledge. Garden City, N.Y.: Praeger.

Bressler, J., Henkin, N., \& Adler, M. (2005). Connecting generations, strengthening communities: Toolkit for intergenerational program planners. Philadelphia, PA: Center for Intergenerational Learning.

Bustillos, A., Fernández Ballesteros, R. y Huici, C. (2012). Efectos de la activación de etiquetas referidas a la vejez. Psicothema, 24(3), 352-357.

Butler, R. (2000). Productive aging: live longer, work longer. Ponencia presentada en el Congreso Mundial sobre Medicina y Salud. Hannover, Alemania. Recuperado de http://www.ilcusa.org/_lib/pdf/Productiv e_Aging.pdf

Caamaño, X. M., \& Martínez, J. L. (2012). La familia como institución intermedia: urdimbre bioafectiva de socialización primaria y espacio de solidaridad intergeneracional. Corintios XIII, 142, 13-43. Recuperado de http://www.caritas.es/imagesrepository/ CapitulosPublicaciones/4382/02-\%20LA\% 20FAMILIA\%20COMO\%20INSTITUCI \%C3\%93N\%20INTERMEDIA.pdf

Cabré, A. (2000). Demografía: una cuestión de dos sexos y cuatro generaciones. Barcelona: Centre d' Estudis Demogràfics.

Carbajo, M. C. (2009). Mitos y estereotipos sobre la vejez. Propuesta de una concepción realista y tolerante. Ensayos: Revista de la Facultad de Educación de Albacete, 24, 87-96. Recuperado de http://www.uclm.es/ab/educacion/ensay os/ensayos24/pdf/24_7.pdf

Centro Latinoamericano y Caribeño de Demografía, CELADE - División de Población de la CEPAL (2007). Informe sobre la aplicación de la Estrategia regional de implementación para América Latina y el Caribe del Plan de Acción Internacional de Madrid sobre el Envejecimiento, Santiago de Chile: Naciones Unidas - CEPAL. Recuperado de http://www.cepal.org/cgi-bin/getprod.as $\mathrm{p}$ ? $\mathrm{xml}=/$ publicaciones $/ \mathrm{xml} / \mathrm{0} / 31980 / \mathrm{P} 3198$ $0 . \mathrm{xml} \& \mathrm{xsl}=/$ celade $/ \mathrm{tpl} / \mathrm{p} 9 \mathrm{f} . \mathrm{xsl} \&$ base $=/ \mathrm{cel}$ ade/tpl/top-bottom.xsl

Comisión Económica para América Latina y el Caribe (CEPAL) (2008). Transformaciones demográficas y su influencia en el desarrollo en América Latina y el Caribe (LC/G.2378 SES.32/14). Santiago de Chile. Recuperado de http://repositorio.cepal.org/bitstream/ha ndle/11362/2894/S0800268 es.pdf;jsession $\mathrm{id}=91 \mathrm{~B} 31 \mathrm{AB} 2 \mathrm{AB} 07908 \mathrm{AC} 34 \mathrm{C} 19841071$ 16DA? sequence $=1$

Comisión Económica para América Latina y el Caribe (CEPAL) (2011). Proyección a largo plazo. Observatorio demográfico, № 11 (LC/ G.2515-P), Santiago de Chile. Recuperado de http://www.cepal.org/es/publicaciones/7 117-proyecciones-poblacion-largo-plazo-lo ng-range-population-projections

Cobo, F., \& Codina, R. (2008). Experiencia práctica: relaciones intergeneracionales y personas mayores. Revista de Educación Social, 8. Recuperado de http://www.eduso. net $/ \mathrm{res} / \mathrm{b}=11 \& \mathrm{c}=99 \& \mathrm{\&}=321$

Delgado, C.R., Calero, C.G., \& González, H. (2016). Potencialidad de los espacios públicos abiertos para las relaciones intergeneracionales: un estudio de caso en la ciudad de Santa Cruz de Tenerife (Canarias, España). Documents d'anàlisi geográfica, 62 (1), 5-25. http://dx.doi.org/10 $.5565 / \mathrm{rev} / \mathrm{dag} .253$

Dulcey, E. (2010). Psicología del envejecimiento y perspectiva del transcurso de la vida: consideraciones críticas. Revista Colombiana de 
Psicología, 19(2), 207-224. Recuperado de http://www.redalyc.org/pdf/804/Resume nes/Resumen_80415435005_1.pdf

Dulcey, E. (2011). Calidad de vida y derecho al reconocimiento en la vejez. Oñate sociolegal series, 1(8), 1-13.

Dulcey, E., Izquierdo, M. E., \& Franco, M. I. (2008). Vivir y envejecer en el Chocó. Revista Javeriana, 742, 72-78.

Dulcey, E., Mantilla, G., García, D. M., \& Alfonso, A. M. (1998). Calidad de vida y previsión de cambios. Bogotá, Colombia: Pontificia Universidad Javeriana y Centro de Psicología Gerontológica.

Eurostat (2012). Informe Envejecimiento activo y solidaridad entre generaciones. Retrato estadístico de la Unión Europea 2012. Recuperado de http://epp.eurostat.ec.euro pa.eu/portal/page/portal/eurostat/home

Eurostat (2014). Eurostat regional yearbook 2014: Population. Recuperado de http://ec.europa.eu/eurostat/documents /3217494/5785629/KS-HA-14-001-EN.P DF/e3ae3b5c-b104-47e9-ab80-36447537e a64

Fernández, A., García, J.L., \& Pérez, F. (2014). Los Programas Universitarios de Mayores y su contribución al aprendizaje a lo largo de la vida. Revista Complutense de Educación, 25(2), 521-540. Recuperado de http://revistas.ucm.es/index.php/RCED/ article/viewFile/42058/42948

Fernández, B. (2012). Participación social entre los adultos mayores chilenos. Revista de Trabajo Social, 83, 61-68. Recuperado de https://dialnet.unirioja.es/servlet/articul o? codigo $=4579328$

Fernández, J. M., \& Kelh, S. (2001). La construcción social de la vejez. Cuadernos de Trabajo Social, 14, 125-162. Recuperado de http://revistas.ucm.es/index.php/CUTS/ article/view/CUTS0101110125A

Fernández-Ballesteros, R. (2008). Envejecimiento activo: la contribución de la Psicología. Madrid: Pirámide.

Fernández-Ballesteros, R. (Ed.) (2009a). PsicoGerontología. Perspectivas europeas para un mundo que envejece. Madrid: Pirámide.
Fernández-Ballesteros, $\quad$ R. (2009b). Envejecimiento activo: contribuciones de la Psicología. Madrid: Pirámide

Fernández-Ballesteros, R., Molina, M. A., Schettini, R., \& Santacreu, M. (2013). The semantic network of aging well. In J. M. Robine, C. Jagger and E. M. Crimmins (Eds.) Healthy Longevity. Annual Review of Gerontology and Geriatrics, 33, New York: Springer.

Fernández Ballesteros, R., Bustillos, A., Huici, C. y Ribera, J. M. (2016). Age discrimination, Eppur Si Muove (Yes It Moves). Journal of the American Geriatrics Society, 64(2), 453-455.

Funes, M. J. (2011). La participación en asociaciones de la población mayor de sesenta y cinco años en España. Análisis de sus efectos e indicaciones para las políticas públicas sectoriales. Revista Internacional de Sociología, 69(1), 167-192.

Galindo, P. (2008). Claves sociológicas para el aprendizaje intergeneracional: dependencia y envejecimiento. En J. A. Valero \& M. Tejedor (coords.). La educación social ante los nuevos retos de la inmigración y los servicios sociales, (pp. 209-224). Valladolid: Servicio de Publicaciones de la Universidad de Valladolid.

Garay, S., \& Hebrero, M. (2015). Los entornos y el envejecimiento en Iberoamérica: análisis a partir de las condiciones de la vivienda. Notas de población. Recuperado de http://reposito rio.cepal.org/handle/11362/39375

García, S., García, R., \& Nieto, M.D. (2013). Programa intergeneracional "Crece Conmigo". En V. Ballesteros (Ed.). Voluntariado y personas mayores (pp. 168-171). Granada: Editorial Universidad de Granada.

Gergen, K. J. (1982). Toward transformation in social knowledge. New York: Springer-Verlag.

Gergen, K. J. (1991). The satured self. Dilemmas of identity in contemporary life. London: Harper.

Gil Calvo, E. (2010). Crisis de madurez y madurez en crisis: el temor a la vejez. Temas para el Debate, 193,19-22. 
Gómez, V. S. (2012). Los procesos de movilidad intergeneracional ascendente en la vida cotidiana familiar. RIPS: Revista de Investigaciones Políticas y Sociológicas, 11(1), 119-140. Recuperado de http://www.redaly c.org/articulo.oa? $\mathrm{id}=38024605006$

González, I. (2010). Moviéndose por el río, programa intergeneracional en Aranda de Duero Sesenta y más, 287, 52-57.

González, M. J., Pedrero, C., Pérez, M. D., \& Prieto, J. (2008). Encuentros intergeneracionales $y$ vida activa en las personas mayores. Un ejemplo: debate intergeneracional sobre violencia de género. En M. C. Palmero (coord.). Formación universitaria de personas mayores y promoción de la autonomía personal. Políticas socioeducativas, metodologías e innovaciones: $X$ Encuentro Nacional de Programas Universitarios para Personas Mayores, (pp. 141-150). Burgos, 5-7 de mayo de 2008.

Goñi, I. (2009). Relaciones intergeneracionales en la dependencia de la población mayor: solidaridad ambivalente y despotismo ilustrado familiar. Sistema: Revista de Ciencias Sociales, 211, 73-90. Recuperado de https://dialnet.unirioja.es/servlet/articul $\mathrm{o}$ ? $\operatorname{codigo}=3016833$

Gracia, E., \& Herrero, J. (2005). Redes sociales de apoyo y ajuste biopsicosocial en la vejez: un análisis comparativo en los contextos comunitario y residencial. Psychosocial Intervention, 14(1), 41-50. Recuperado de http://www.redalyc.org/articulo.oa?id= 179817564003

Guillén, F. (1992). La prevención y la enfermedad en las personas mayores. En J. L. Pinillos. Una aproximación pluridisciplinar al entorno de la vejez (pp. 69-78). Barcelona: S.G. Editores.

Hank, K. (2012). Relaciones entre generaciones en Europa: una panorámica de las diferentes dimensiones de solidaridad familiar intergeneracional. Panorama Social, 15, 9-22. Recuperado de https://dialnet.uni rioja.es/servlet/articulo?codigo $=3987808$

Harwood, J., McKee, J., \& MeiChen, L. (2000). Younger and older adult schematic representation of intergenerational communication. Communication Research, 67, 20-41. http:// dx.doi.org/10.1080/03637750009376493

Hatton-Yeo, A. (2007). Programas intergeneracionales, solidaridad intergeneracional y cohesión social. En M. Sánchez (coord.). Programas intergeneracionales: hacia una sociedad para todas las edades, (pp. 123-140). Barcelona: Obra Social de La Caixa.

Herce, J. A. (2016). El impacto del envejecimiento de la población en España. Cuadernos de Información Económica, 251, 39-48. Recuperado de https://dialnet.unirio ja.es/servlet/articulo? codigo $=5497234$

Herrera, M., Sani, F., \& Bowe, M. (2011). Perceived family continuity: implications for family identification and psychological well-being. Revista de Psicología Social, 26(3), 387-399. http://dx.doi.org/10.1174/ 021347411797361275

Huenchan, S. (2016). Envejecimiento e institucionalidad pública en América Latina y el Caribe: conceptos, metodologías y casos prácticos. Recuperado de http://rep ositorio.cepal.org/handle/11362/40197

Iacub, R. (2002). La postgerontología: hacia un renovado estudio de la gerontología. Revista Latinoamericana de Psicología, 34(1-2), 155-157. http://www.redalyc.org/pdf/805/8 0534212.pdf

Ibáñez, T. (1994). Psicología Social Construccionista. México: Dirección de Publicaciones de la Universidad de Guadalajara.

Ibáñez, T. (1990). Aproximaciones a la psicología social. Barcelona: Senday.

Ibáñez, J. (1985). Del algoritmo al sujeto. Perspectivas de la investigación social. Madrid: Siglo XXI.

Igartua, M.T. (2016). Cambio demográfico: envejecimiento de la población, prolongación de la vida activa y Seguridad y Salud en el trabajo. Revista Internacional y Comparada de Relaciones Laborales y Derecho del Empleo, 4(1), 152-184. 
Recuperado de https://idus.us.es/xmlui/han dle/11441/46375

Instituto Nacional de Estadística I.N.E. (2011). Censo de población y vivienda 2011. Madrid: Ministerio del Interior.

International Longevity Centre Brazil ILC-Br (2015). Active Ageing: A policy framework in response to the longevity revolution. Brasil: International Longevity Centre Brazil.

Izquierdo, G. (2008). Envejecimiento y vejez. Revista Javeriana, 742, 3-6. Recuperado de https://dialnet.unirioja.es/servlet/articul $\mathrm{o}$ ? codigo $=2555868$

Jones, F. (2016). Ageing in the Caribbean and the human rights of older persons: twin imperatives for action. CEPAL. Studies and Perspectives - ECLAC Subregional Headquarters for The Caribbean. Recuperado de http:// www.cepal.org/fr/node/35882

Lemus, S., \& Expósito, F. (2005). Nuevos retos para la Psicología Social: edadismo y perspectiva de género. Pensamiento psicológico, 5, 34-51. Recuperado de http://www.redalyc.org/pdf/ 801/80112046005.pdf

Llaneza, P. (2010). ¿A qué jugaban mis abuelos?: galardón a las buenas prácticas. Revista Asturiana de Terapia Ocupacional, 8, 14-21. Recuperado de https://dialnet.unirioja.es/ servlet/articulo? codigo $=3823384$

Llorente, X. (2012). Año Europeo 2012 Envejecimiento activo y solidaridad intergeneracional. Educación social: revista de intervención socioeducativa, 51,11-26. Recuperado de https://dialnet.unirioja.es/se rvlet/articulo? codigo $=4075134$

López, B. (2012). Empoderamiento y adultos mayores. Impacto de la participación de un grupo de adultos mayores en un programa educativo. En Envejecimiento en América Latina y el Caribe. Enfoques interdisciplinarios en Investigación y docencia de la Red Latinoamericana de Investigación en Envejecimiento (LARNA). México: Instituto de Investigaciones Sociales, Universidad Nacional Autónoma de México (UNAM).
López, G. (2012). Repensar el Estado de bienestar: la sostenibilidad del bienestar intergeneracional en España y la OCDE. Ekonomoaz: Revista vasca de economía, 81, 16-37. Recuperado de https://www.upf.edu/ documents/2911971/2939989/Ekonomiaz 2012.pdf/a9ca2b7e-b029-4148-8a01-309c Oaba010c

López-Calleja, C., \& Morejón, B. (2015). Envejecimiento demográfico y migración interna de personas con 60 años y más de edad en Cuba y países de América latina. Población y Salud en Mesoamérica, 12(2). Recuperado de http://revistas.ucr.ac.cr/ind ex.php/psm/article/view/17381/20010

López Doblas, J., \& Díaz, M. P. (2013). La modernización social de la vejez en España. Revista Internacional de Sociología, 71(1), 65-89. http://dx.doi.org/10.3989/ris.2011.0 4.26

López-Pastor, C. (2014). El proceso de envejecimiento: una revisión bibliográfica sobre la influencia de la participación social sobre la salud y la calidad de vida de las personas mayores. Trabajo Social y Salud, 79, 83-89. Recuperado de https://dialnet.unirio ja.es/servlet/articulo?codigo $=4909547$

Lucas, B., Pérez, A., Fonseca, E., \& Ortuño, J. (2015). Programas educativos universitarios para mayores: Evaluación de su Impacto en la Autopercepción del Apoyo Social y la Salud Mental. Revista Colombiana de Psicología, 24(1), 47-60. http ://dx.doi.org/10.15446/rcp.v24n1.43437

Martínez del Castillo, J., Jiménez-Beatty, J. E., Graupera, J. L., \& Rodríguez, M. L. (2006). Condiciones de vida, socialización y actividad física en la vejez. Revista Internacional de Sociología, 64(44), 39-62. h ttp://dx.doi.org/10.3989/ris.2006.i44.27

Meil, G. A. (1999). La postmodernización de la familia española. Madrid: Acento.

Meil, G. A. (2004). Cambios en las relaciones familiares $y$ en la solidaridad familiar. Revista Española Arbor, CLXXVIII, 263-312. Recuperado de http://www.uam.es/personal_pdi/econo 
micas/gmeil/espaniol/publicaciones/solidari dadfam2004.pdf

Melero, M. (2007). Modificaciones de los estereotipos sobre los mayores. En L. Alvarez, J. Evans y B. Kristensen (comps.). Comunicación Actas do Foro Internacional e Persoas Maiores: Actas do Foro Internacional (pp. 29-46). Colexio Profesional de Xornalistas de Galicia.

Minois, G. (1989). Historia de la vejez. De la antigüedad al Renacimiento. Madrid: Nerea.

Miralles, I. (2010). Vejez productiva: el reconocimiento de las personas mayores como un recurso indispensable en la sociedad. Cairos: Revista de Temas sociales, 26. Recuperado de https://dialnet.unirioja. es/servlet/articulo? codigo $=3702472$

Molina, S., Martínez, G., Bordes, M., \& López Piñero, M. (2015). Prácticas sociales de personas mayores en el ámbito universitario: el Voluntariado Social como estrategia para la inclusión. Margen: revista de Trabajo Social y Ciencias Sociales, 77. Recuperado de http://www.margen.org/sus cri/margen77/voluntariado.pdf

Monserud, M. A. (2008). Intergenerational relationships and affectual solidarity between grandparents and young adults. Journal of Marriage and Family. 70(1), 182195. http://dx.doi.org/10.1111/j.1741-3737 .2007.00470.x

Moody, H. R. (2010). Aging: concepts and controversies (6a ed.). Thousand Oaks, California: Sage Publications.

Moral, M. V. (1999). La sociedad hace viejos a nuestros mayores. Ponenciapresentada en el III Congreso Hispano Luso sobre las Personas Mayores. Cáceres, España.

Moral, M. V., \& Ovejero, A. (2004). Jóvenes, globalización y postmodernidad: Crisis de la adolescencia social en una sociedad adolescente en crisis. Papeles del Psicólogo, 25(87), 72-79. Recuperado de http://www. redalyc.org/pdf/778/77808710.pdf

Morin, E. (1983). Interdisciplinariedad y ciencias humana. Madrid: Tecnos/UNESCO.

Naciones Unidas (2014). La situación demográfica en el Mundo,
2014. Informe conciso. Recuperado de http://www.un.org/en/development/des a/population/publications/pdf/trends-repor t.pdf

Naciones Unidas (2011). World Population Prospects: the 2010 revision. Nueva York. Recuperado de http://esa.un.org/unpd/wpp /index.htm

Naciones Unidas-Centro Latinoamericano y Caribeño de Demografía (CELADE)

-División de Población Comisión Económica para América Latina y el Caribe (CEPAL) (2011). Los derechos de las personas mayores. Materiales de estudio y divulgación. Paradigma sobre el envejecimiento y la vejez, 2011. Recuperado de http://www.cepal.org/celade/noticias/do cumentosdetrabajo/2/43682/Modulo_1.pdf

Naciones Unidas (2010). World Population Ageing 2009 (ST/ESA/ SER.A/295). Nueva York. Recuperado de http://www.un.org/esa/population/publi cations/WPA2009/WPA2009-report.pdf

Naciones Unidas-CEPAL (2009). El envejecimiento y las personas de edad. Indicadores sociodemográficos para América Latina. Santiago de Chile: Naciones Unidas.

Neri, A. L. (2007). El legado de Paul B. Baltes a la Psicología: el paradigma lifespan aplicado al desarrollo y al envejecimiento. Revista Latinoamericana de Psicología, 39(2), 413-417. Recuperado de http://www.redaly c.org/pdf/805/80539214.pdf

Organización Mundial de la Salud (OMS) (2015). Diez datos sobre el envejecimiento y la salud. Recuperado de http://www.who. int/features/factfiles/ageing/es/

Organización Iberoamericana de Seguridad Social OISS (2008). Personas Mayores, Dependencia y Servicios Sociales en los países del Cono Sur. OISS.

Padrós, C., Moll, E., \& Gancedo, F.R. (2009). Programa intergeneracional: "Gent gran. Gen petit: una experiencia compartida". Anuari de l'envelliment, 241-260. Recuperado 
de http://ibdigital.uib.es/greenstone/collect /anuariEnvelliment/archives/2009_anu/ari _enve/liment_/p241.dir/2009_anuari_env elliment_p241.pdf

Pàmies, T. (2002). La aventura de envejecer. Barcelona: Península.

Pérez, R., \& Smith, V. (2008). Comunicación intergeneracional: el cuestionario de percepción de la comunicación intergeneracional $(\mathrm{CPCI})$ en el contexto costarricense. Revista Interamericana de Psicología, 42(3), 589-603. Recuperado de http://www.psicorip.org/Resumos/PerP/ RIP/RIP041a5/RIP04258.pdf

Pérez Serrano, G. (2012). Envejecimiento activo y solidaridad intergeneracional: claves para un envejecimiento activo. Madrid: Universidad Nacional de Educación a Distancia, UNED.

Petretto, D. R., Pili, R., Gaviano, L., Matos, C., \& Zuddas, C. (2016). Envejecimiento activo y de éxito o saludable: una breve historia de modelos conceptuales. Revista Española de Geriatría y Gerontología, 51(3), 127-186. Recuperado de https://dialnet.unirioja.es/se rvlet/articulo? codigo $=5661398$

Pinazo, S. (2012). Las personas mayores proveedoras de conocimientos y cuidados. El papel de los programas intergeneracionales. Educación Social: Revista de Intervención Socioeducativa, 51, 45-66. Recuperado de http://www.raco.cat/index.php/Educaci oSocial/article/download/260501/369076

Pinazo, S., \& Montoso, J. (2004). La relación entre abuelos y nietos: factores que predicen la calidad de la relación intergeneracional. Revista Internacional de Sociología, 38, 147-168. http:// dx.doi.org/10.3989/ris.2004.i38.257

Pinazo, S., \& Poveda, R. (2016). Innovación para el envejecimiento activo en la Unión Europea. Análisis del programa "Ambient assisted living joint programme" (AAL) en el periodo 2008-2015. Búsqueda, 15, 38-50 Recuperado de http://revistas.cecar.edu.co/ busqueda/article/download/95/88

Pinillos, J. L. (1992). Realidades y mitos de la tercera edad. En Perspectivas en Gerontología y Salud (pp. 102-115). Valencia: Promolibro.

Pinheiro, M. C., Koller, M. E., \& Sánchez-Soares, P. (2008). Adaptación y uso de una medida de evaluación de la red de apoyo social — diagrama de escolta- para personas mayores en Brasil. Universitas Psychologica, 7(2), 493-505.

Population Reference Bureau (2007). 2007-World Population Data Sheet, Washington D.C.: PRB.

Population Reference Bureau (2009). Cuadro de datos de la población mundial - 2009 [en línea]. Recuperado de http://www.prb.org/ pdf09/09wpds_sp.pdf

Rice, C. J., Löckenhoff, C. E., \& Carstensen, L. L. (2002). En busca de independencia y productividad: cómo influyen las culturas occidentales en las explicaciones individuales y científicas del envejecimiento. Revista Latinoamericana de Psicología, 34(1-2), 133-154. Recuperado de http://www.redalyc.org/pdf/805/805342 11.pdf

Riegel, F. K., \& Meacham, J. A. (eds.) (1976a). The developing individual in a changing world: I. Historical and cultural issues. Chicago, EE.UU.: Aldine.

Riegel, F. K., \& Meacham, J. A. (eds.) (1976b). The developing individual in a changing world: I. Historical and cultural issues: II. Social and environmental issues. Chicago, EE.UU.: Aldine.

Rodríguez, E. (2009). Envejecimiento activo. Agathos: Atención sociosanitaria y bienestar, 9(4), 42-49. Recuperado de https://dialnet.unirioja.es/servlet/articul $\mathrm{o}$ ? codigo $=3166143$

Rodríguez, A., Gutiérrez, M.C., \& Seijas, M.B. (2012). Envejecimiento activo, aprendizaje a lo largo de la vida y buenas prácticas en los programas universitarios para las personas mayores. En G. Pérez Serrano (Coord.). Envejecimiento activo y solidaridad intergeneracional: claves para un envejecimiento activo (p. 64). Madrid: Universidad Nacional de Educación a Distancia, UNED. 
Romero, I., Serrano, M.I., \& Godoy, I. (2015). Cómo afecta en la salud de los abuelos canguro el cuidado de sus nietos. En J. J. Gázquez (Coord). Calidad de vida, cuidadores e intervención para la mejora de la salud en el envejecimiento (pp. 145-150). Almería: Asociación Universitaria de Educación y Psicología.

Ruiz, I., \& Vicente, F. (2006). Programa intergeneracional para la recuperación de tradiciones y costumbres populares. En V. Pérez, J. L. Alagón y L. V. Amador (coords.). Vejez, autonomía o dependencia, pero con calidad de vida (pp. 139-150). Sevilla: Dykinson.

Ruiz, M, V., Scipioni, A M., \& Lentini, D. F. (2008). Aprendizaje en la vejez e imaginario social. Fundamentos en Humanidades, IX(17), 221-233. http://www .redalyc.org/pdf/184/18417111.pdf

Sánchez, C., Trianes, M, V., \& Blanca, M. J. (2009). Estereotipos negativos hacia la vejez y su relación con variables sociodemográficas en personas mayores de 65 años. Revista Española de Geriatría y Gerontología: Órgano oficial de la Sociedad Española de Geriatría y Gerontología, 44, 124-129.

Sánchez, M., Buz, J., Bueno, M. B., \& Navarro, A. B. (2007). Abuelas, abuelos... y nietos: vinculación intergeneracional. En F. López (coord.). La escuela infantil: observatorio privilegiado de las desigualdades sociales, (pp. 183-194). Barcelona: Grao.

Sánchez-Oro, M., Pérez, J. A., \& Moreno, J. (2013). Los mayores en el continuo rural-urbano. Aproximación a la percepción subjetiva y expectativas vitales (el caso de Extremadura). Papers, 98(1), 143-174. Recuperado de https://ddd.uab.cat/pub/papers/papers_a 2013m1-3v98n1/papers_a2013m1-3v98n1 p143.pdf

Sánchez-Vera, P., Algado, M. T., Centelles, F., López, J., \& Jiménez, B. (2009). Viudedad y vejez. Estrategias de adaptación a la viudedad de las personas mayores en España. Valencia: Nau Llibres.
Sancho, P., Tomás, J.M., Gutiérrez, M., Oliver, A., \& Galiana, L. (2015). Estabilidad de la satisfacción vital y su relación con la salud y el bienestar subjetivo en el adulto mayor. En J. J. Gázquez (Coord). Calidad de vida, cuidadores e intervención para la mejora de la salud en el envejecimiento (pp. 191-197). Almería: Asociación Universitaria de Educación y Psicología.

Scheibe, S., \& Carstensen, L. L. (2010). Emotional aging: recent findings and future trends. Journal of Gerontology: Psychological Sciences, 65B(2), 135-144. https://doi.org/1 $0.1093 /$ geronb/gbp132

Serrani, D. (2010). Resolución de dilemas reales en adultos mayores. Estudio comparativo en una comunidad argentina. Revista de Geriatría y Gerontología, 45(6), 326-330. Recuperado de https://www.academia.edu/ 1390929/Resoluci\%C3\%B3n de dilemas reales en adultos mayores. Estudio com parativo_en_una_comunidad_argentina

Stenner, P., McFarquhar, T., \& Bowling, A. (2011). Older people and active ageing: subjective aspects of ageing actively. Journal of Health Psychology, 16(3), 467. http://dx.d oi.org/10.1177/1359105310384298

Triadó, C. y Osuna, M.J. (2005). Las relaciones entre abuelos y nietos. En Pinazo, S. y Sánchez, M. (Eds.), Gerontología: actualización, valoración y propuestas. Madrid: Pearson-Prentice Hall.

Trujillo, S. (2005). Agenciamiento individual y condiciones de vida. Universitas Psychologica, 4(2), 221-229. Recuperado de http://www.scielo.org.co/pdf/rups/v4n2/ v4n2a09.pdf

Trujillo, S., Tovar, C., \& Lozano, C. (2007). El anciano conformista iun optimista con experiencia?: sobre lo psicológico en la calidad de vida con personas mayores en Soacha y Sibaté. Universitas Psychologica, 6(2), 263-268. Recuperado de http://www. scielo.org.co/pdf/rups/v6n2/v6n2a06.pdf

Uprimny, M. (2010). Perspectivas y propuestas sobre Política Pública. En Diálogos sobre envejecimiento. Mesa de 
trabajo sobre envejecimiento y vejez en Colombia. Fundación Cepsiger para el Desarrollo Humano y Confederación Colombiana de ONG, 19-30. Recuperado de http://fundacioncepsiger.org/nuevosite/ wp-content/uploads/2011/11/Di\%C3\%A11 ogos-sobre-envejecimiento-y-vejez.pdf .

Vila, J. M. (2012). La educación en el envejecimiento activo y la solidaridad intergeneracional. VII jornadas de la Asociación Estatal de Programas Universitarios para Mayores. Sesenta y más, 231, 32-33.

Williams, A., \& Garrett, P. (2002). Communication evaluations across the life span. From adolescent storm and stress to elder aches and pains. Journal of Language and Social Psychology, 21(2), 101-126. Recuperado de http://journals.sagepub.com/doi/abs/10. 1177/0261927X02021002001?legid=spjls \%3B21\%2F2\%2F101\&patientinform-links $=$ yes

\section{Notas}

* Artículo de investigación 